\title{
Osteochondral Flaps from the Distal Femur: Expanding Applications, Harvest Sites, and Indications
}

\author{
James P. Higgins, MD ${ }^{1}$ Heinz K. Bürger, MD² \\ ${ }^{1}$ Curtis National Hand Center, Baltimore, Maryland \\ 2 Private Hospital Maria Hilf, Klagenfurt, Austria \\ J Reconstr Microsurg 2014;30:483-490.
}

\begin{abstract}
Address for correspondence James P. Higgins, MD, Care of Anne Mattson, The Curtis National Hand Center, MedStar Union Memorial Hospital, 3333 North Calvert Street, JPB Mezzanine, Baltimore, MD 21218 (e-mail: anne.mattson@medstar.net).
\end{abstract}

\begin{abstract}
Keywords

- descending geniculate artery

- Kienböck disease

- medial femoral condyle

- medial femoral trochlea flap

- osteocartilagenous autograft

- scaphoid nonunion

- vascularized bone
\end{abstract}

Background The medial and lateral femur provide a source of convex osteochondral vascularized bone. The medial trochlea has been demonstrated to have similar contour to the proximal scaphoid, lunate, and capitate. Other sites of osteochondral harvest such as the posteromedial femur and the lateral trochlea are similar in morphology to the humeral capitellum and medial talus, respectively. These analogous structures offer potential solutions to difficult articular problems.

Patients and Methods Patients who underwent osteochondral reconstruction of the extremities were reviewed. These included 16 medial femoral trochlea (MFT) scaphoid nonunion reconstructions, 16 MFT Kienböck lunate reconstructions, 5 MFT capitate reconstructions, 2 lateral femoral trochlea osteochondral reconstructions of medial tarsal avascular necrosis, and 5 posteromedial femoral osteochondral reconstructions of the humeral capitellum for posttraumatic arthritis.

Results Computed tomography (CT) imaging demonstrated 15 of 16 reconstructed scaphoids achieving union. Follow-up wrist motion averaged 46.0 degrees extension and 43.8 degrees flexion, similar to preoperative measurements. CT imaging confirmed healing in 15 of 16 reconstructed lunates. Lichtman staging remained unchanged in 10 patients, improved in 4 patients ( $3 A-2)$, and worsened in 2 patients (3A-3B). All but one patient experienced improvement in wrist pain. Wrist range of motion at follow-up averaged 50 degrees extension and 38 degrees flexion, similar to preoperative measurements. Capitate, talar, and capitellar reconstructions all resulted in achievement of osseous healing and restoration of joint alignment.

Conclusions Vascularized osteochondral flaps provide a useful tool in the treatment of difficult articular problems in the extremities. Clinical experience thus far demonstrates a high rate of achieving union with acceptable range of motion and good pain relief.
The vascular tree of the descending geniculate artery (DGA) is the source vessel for a large number of reconstructive flap options. Its numerous branches have led to the description of free flaps providing skin, tendon, periosteal, corticoperiosteal, corticocancellous, and osteochondral flaps. The DGA vascular anatomy has been well described and has led to an increased understanding of the small vessel anatomy surrounding the distal femur. ${ }^{1-4}$

The DGA vascular system provides a dense filigree of blood vessels invested in the periosteum of the medial column of the distal femur. The DGA divides into two major periosteal branches: the longitudinal branch and the transverse branch. received

December 12, 2013

accepted after revision

January 11, 2014

published online

May 6, 2014
Copyright $\odot 2014$ by Thieme Medical Publishers, Inc., 333 Seventh Avenue, New York, NY 10001, USA. Tel: +1(212) 584-4662.
DOI http://dx.doi.org/ 10.1055/s-0034-1372484. ISSN 0743-684X. 
The longitudinal branch, which courses to the condyle, is used as the source vessel for the corticoperiosteal flap commonly used for extra-articular nonunions. The transverse branch, which courses anteriorly, is invested in the periosteum surrounding the medial femoral trochlea (MFT). ${ }^{3,4}$ Hugon et al have observed that this site is a source of a convex cartilaginous flap with a curvature similar to that of the proximal scaphoid as well as the concave surface of the scaphoid fossa. ${ }^{4}$ Bürger et al reported early experience with the use of this segment of bone and cartilage as a vascularized flap for scaphoid proximal pole reconstructions ${ }^{5}$ and lunate reconstruction in advanced Kienböck disease, ${ }^{6}$ with further studies underway.

The authors have also developed early experience with other novel osteochondral flap options from this region. These include the following:

- The use of the DGA as a source vessel for osteochondral flaps from the posterior medial femur.

- The use of the DGA as a source vessel for composite osteochondral flaps from the MFT and corresponding proximal medial patella via vessel branches within the joint capsule.

- The superolateral geniculate artery (SLGA) as a source vessel for osteochondral flaps from the lateral femoral trochlea.

This installment will serve as an invited review of these early experiences as presented in the 2013 WSRM panel "Flaps, Flaps, Flaps." The focus will be on the applications in scaphoid and lunate reconstruction where our experience is most robust and data will be provided.

\section{Scaphoid Reconstruction}

Conventional treatment options for proximal pole scaphoid nonunions include achievement of ridged internal fixation with bone grafting, local vascularized pedicle bone flaps, or microvascular free bone flaps.

The recalcitrant nonunion of the proximal pole will often present with a very small and poor quality proximal segment after multiple previous surgical fixation attempts. It is in this setting that conventional surgical treatment options provide inadequate solutions for the absence or poor quality of proximal pole cartilage. The ability to provide a cartilagebearing flap of similar contour to the convex scaphoid proximal pole enables the surgeon to resect the necrotic and diminutive proximal pole and insert a well vascularized and congruent flap (-Fig. 1). The proximal pole is purposefully resected distally to the level of the scaphoid waist so that a large flap segment may be accommodated and satisfying screw fixation obtained between the flap and the distal native distal pole of the scaphoid.

The use of this segment of bone and cartilage as a vascularized flap for proximal scaphoid reconstruction was initially described by the senior author (H.K.B.) in a case report in 2008. ${ }^{7}$ Subsequently, these authors have performed collectively a series of 55 proximal pole osteochondral scaphoid reconstructions. Their early experience in the use of this flap in the initial 16 patients was reported resulting in good intermediate term results at a minimum of 6 months followup. ${ }^{5}$ This published study also provides a detailed description of the operative technique. A summary of the technique and results of that study follows.

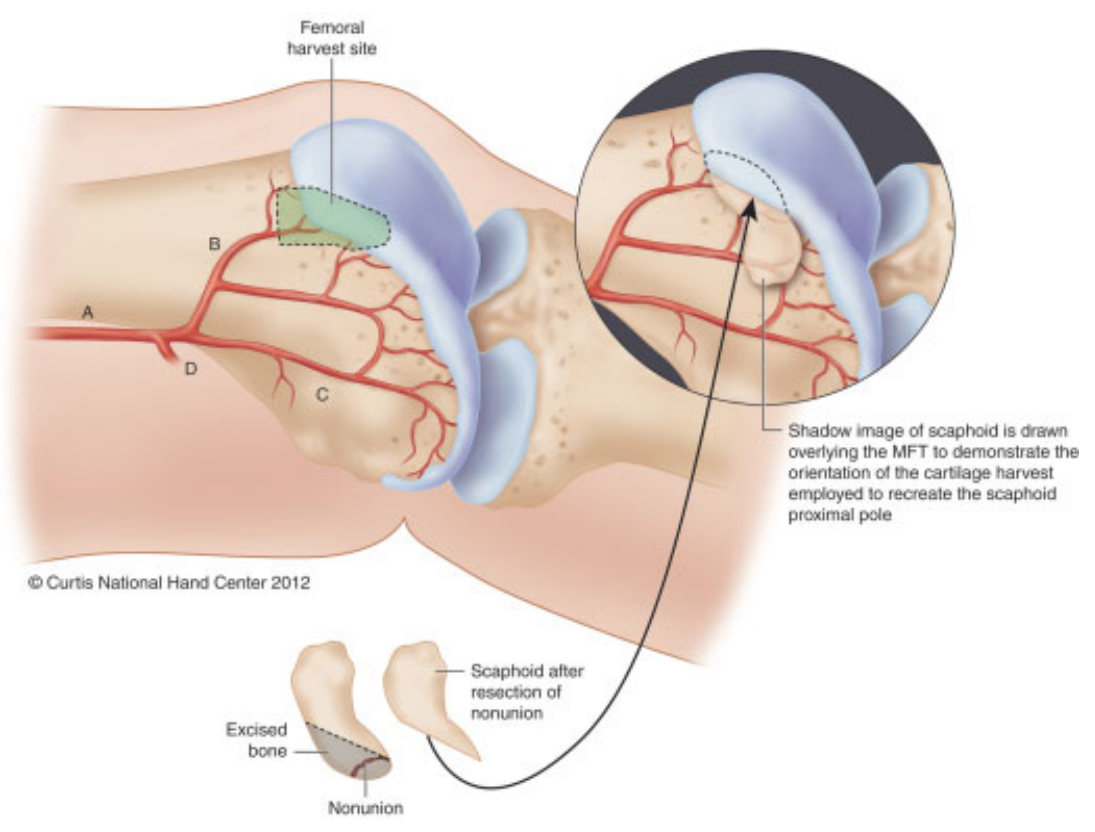

Fig. 1 Representation of medial femoral trochlea (MFT) and the planned portion of reconstructed proximal scaphoid. Portion of MFT harvested to provide vascularized osteocartilaginous reconstruction of the proximal scaphoid. A, descending geniculate artery; B, transverse branch; $C$ longitudinal branch; D, superomedial geniculate artery. (Source: Bürger HK, Windhofer C, Gaggl AJ, Higgins JP. Vascularized medial femoral trochlea osteocartilaginous flap reconstruction of proximal pole scaphoid nonunions. J Hand Surg Am 2013;38(4):690-700.) 


\section{Surgical Technique}

The surgical approach for harvesting medial femoral condyle (MFC) and MFT flaps through the soft tissue is identical. The authors have described this surgical approach including the potential use of a skin segment of the flap in its harvesting technique previously. ${ }^{5,8,9}$ A portion of these descriptions is provided here.

The DGA can be traced distally along the medial column of the femur to feed a broad filigree of blood vessels intimately adherent into the periosteum of the medial distal femur. The longitudinal branch supplies the region commonly used for harvest of the MFC osteoperiosteal flap. The perpendicular transverse branch typically traverses the metaphyseal region and densely supplies the periosteum surrounding the MFT both on its medial aspect and proximal aspect. This is the vessel that is used for MFT osteochondral reconstruction.

After ligating the DGA from the superficial femoral artery, the DGA is reflected distally. The longitudinal branch to the condyle may be ligated. Harvest continues using the transverse branch that can be traced toward the medial trochlea. The periosteal vessels are left adherent to the territory of bone and cartilage desired for harvest. A sagittal saw and osteotomes may be used to harvest the proximal most portion of the cartilage-bearing segment of the MFT. The dimensions correspond as follows:

- The proximal-to-distal harvest is measured to recreate the radial-to-ulnar dimensions of the defect in the wrist.

- The anterior-to-posterior harvest is measured to recreate the proximal-to-distal dimension of the defect in the wrist.

- The medial-to-lateral harvest in the knee is measured to recreate the volar-to-dorsal dimensions of the wrist.

The osteocartilaginous segment is then fashioned so as to fit into the defect created from resection of the proximal scaphoid. The convex surface of the flap effaces the concave scaphoid fossa of the radius. The cancellous portion effaces the adjacent lunate as well as the preserved distal cartilaginous shell of the lesser curvature of the native scaphoid. A bone segment is usually affixed with a single cannulated screw inserted from the dorsal or volar approach (surgeon's preference).

\section{Summary of Case Series Results ${ }^{5}$}

Healing in the cohort of 16 consecutive osteocartilaginous scaphoid arthroplasties was confirmed by CT scan in 15

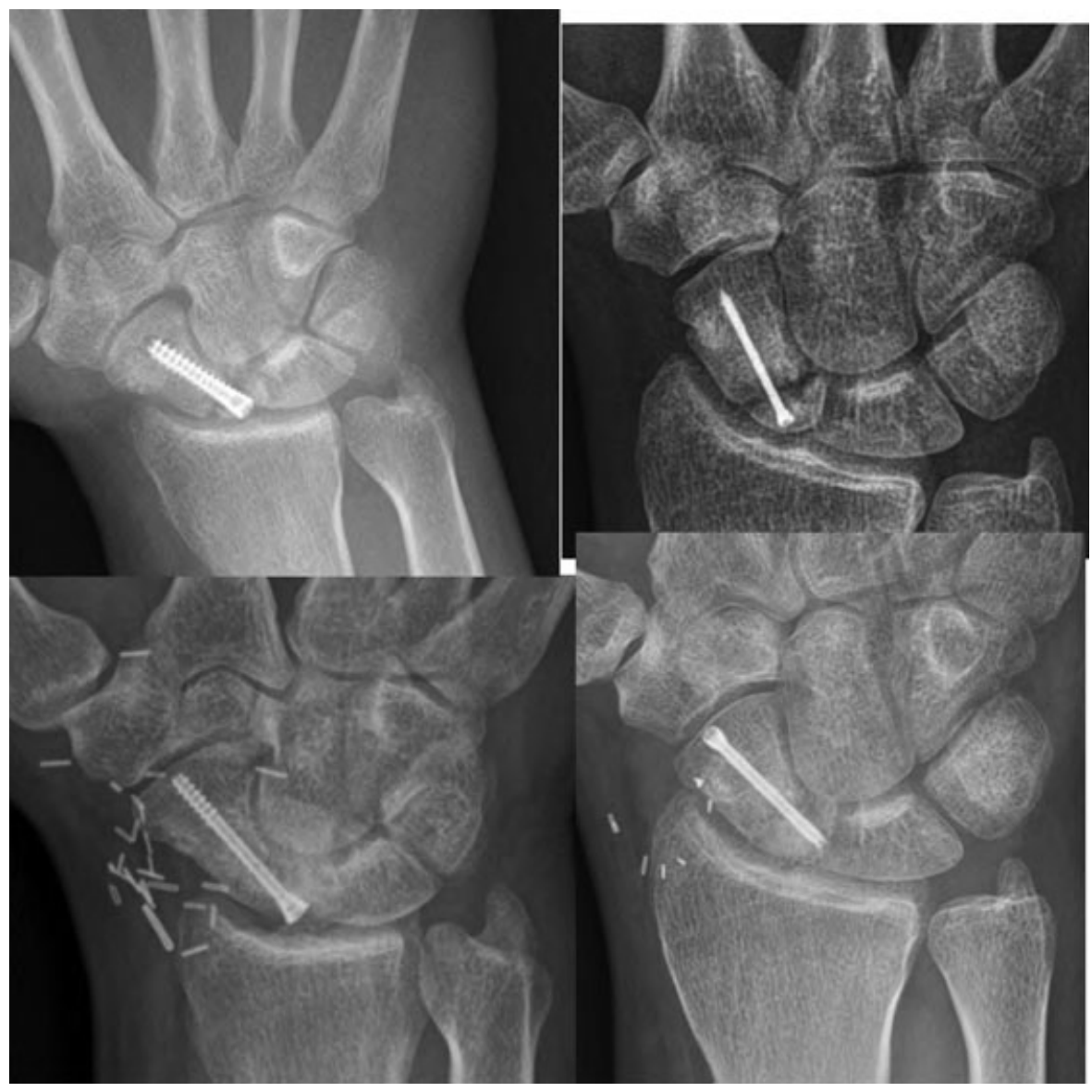

Fig. 2 Preoperative anteroposterior (AP) (above) and postoperative AP and lateral X-rays (below) of cases of scaphoid nonunion medial femoral trochlea reconstructions. X-rays on the left demonstrate a case using a dorsal approach showing preoperative nonunion (above) and postoperative healed reconstruction (below) in a 1-year postoperative X-ray. X-rays on the right demonstrate a case using a volar approach showing preoperative nonunion (above) and postoperative healed reconstruction (below) 1-year postoperative X-ray. 


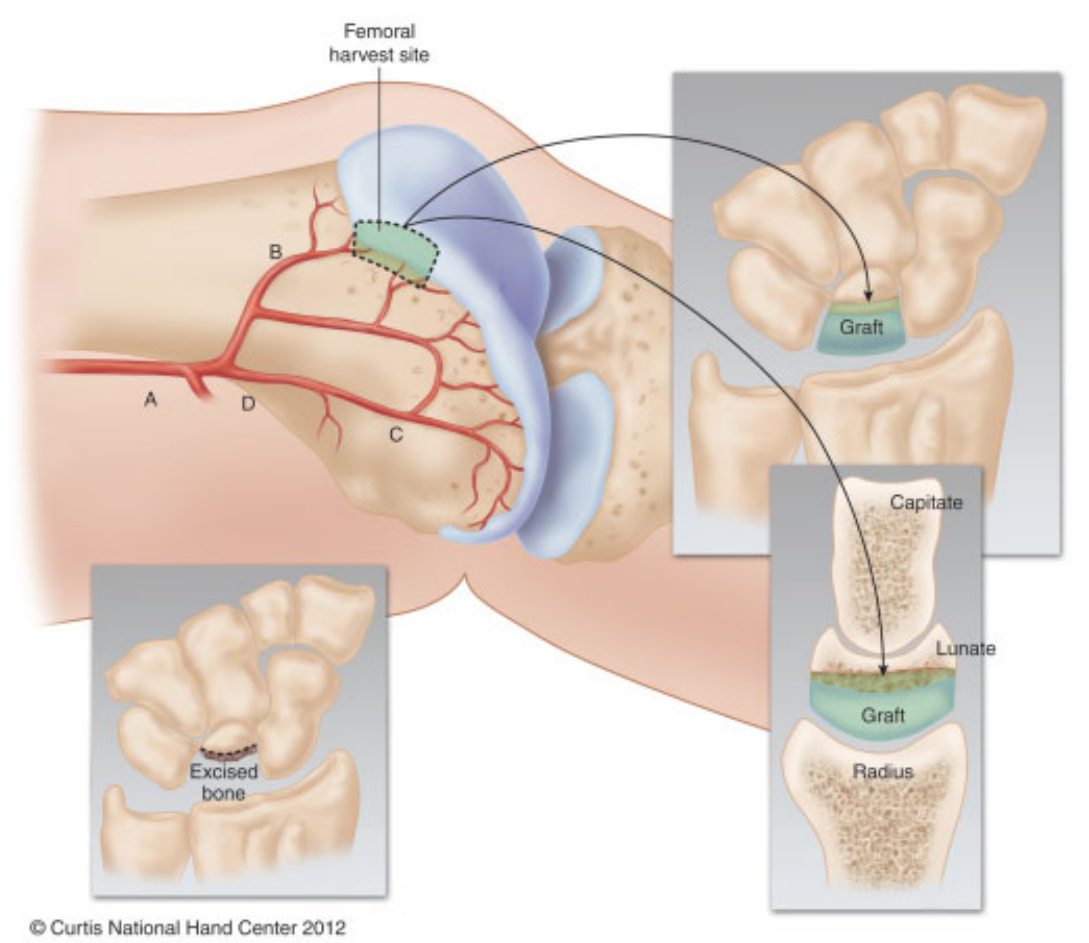

Fig. 3 Representation of medial femoral trochlea (MFT) and the planned portion of reconstructed proximal lunate portion of MFT harvested to provide vascularized osteocartilaginous reconstruction of the proximal lunate. Note the preservation of the native cartilage effacing the midcarpal joint. Vascular anatomy demonstrates branching pattern and supply of trochlea periosteum. A, descending geniculate artery; B, transverse branch to MFT; C, longitudinal branch; D, superomedial geniculate artery.

patients. In one patient, healing was not achieved and the patient is considering salvage operations.

A minimum of 6 months follow-up was required for inclusion in this series. The average follow-up was 14 months with a range from 6 to 72 months. The median age of the patients was 30 years with the range of 18 to 47 years. Average number of previous operations was one with the range of zero to three. Of 16 patients, 7 were smokers.

The surgical technique varied for the 16 , with 13 of 16 being approached volarly and the remaining 3 being approached dorsally. Six cases were treated with cannulated screw fixation, 4 with screw and miniplate combination, 3 with miniplate and K-wire, and 2 with $\mathrm{K}$-wires alone, and 1 with miniplate alone.

Of 16 patients, 12 reported complete pain relief whereas 4 of 16 reported improvement without complete pain relief. Pronosupination and digital range of motion were unaffected when comparing preoperative to postoperative values. Preoperative extension averaged 45.7 degrees, and postoperative extension averaged 46 degrees. Preoperative flexion averaged 43 degrees, and postoperative flexion averaged 43.8 degrees.

Radiographically, the analysis of the intercarpal relationships demonstrated relatively unchanged scapholunate angles with an average of 51.6 degrees preoperatively and 48.6 degrees postoperatively (-Fig. 2 ).

\section{Lunate Reconstruction}

Cadaveric studies have described the pertinent vascular arcade supplying this bone and cartilage $e^{3,4}$ and the anatomic similarities between the greater curvature of the proximal lunate with the MFT. ${ }^{4}$

In the setting of advanced Kienböck disease, conventional treatment has known limitations. More conventional joint leveling procedures are reserved for less advanced disease where lunate architecture is preserved. In the setting of stage 2 Kienböck disease with early collapse of the radial side of the lunate, or stage 3 Kienböck disease with complete collapse of the lunate, if lunate-sparing techniques are pursued, conventional surgery requires use of pedicled vascularized corticoperiosteal bone from the distal radius after reexpansion of the fractured cartilaginous shell of the lunate. This requires reliance on the native avascular cartilage. The availability of osteocartilaginous vascularized bone provides a solution to the vascularity of the lunate and also replaces the loadbearing cartilage of the radiocarpal articulation while employing only the (often less damaged) midcarpal native lunate cartilage. This enables the surgeon to resect the entire proximal lunate, preserving only the midcarpal articulating distal wafer of native lunate cartilage. The MFT flap provides the convex surface of cartilage that articulates with the lunate fossa of the radius (-Fig. $\mathbf{3}$ ).

The authors have thus far performed collectively a series of 30 proximal osteochondral lunate reconstructions. An institutional review board approved study on the outcomes in the initial 16 patients with a minimum of 1 -year follow-up is underway. This published study will also provide a detailed description of the operative technique. A qualitative summary of the evolving results of that study follows. 


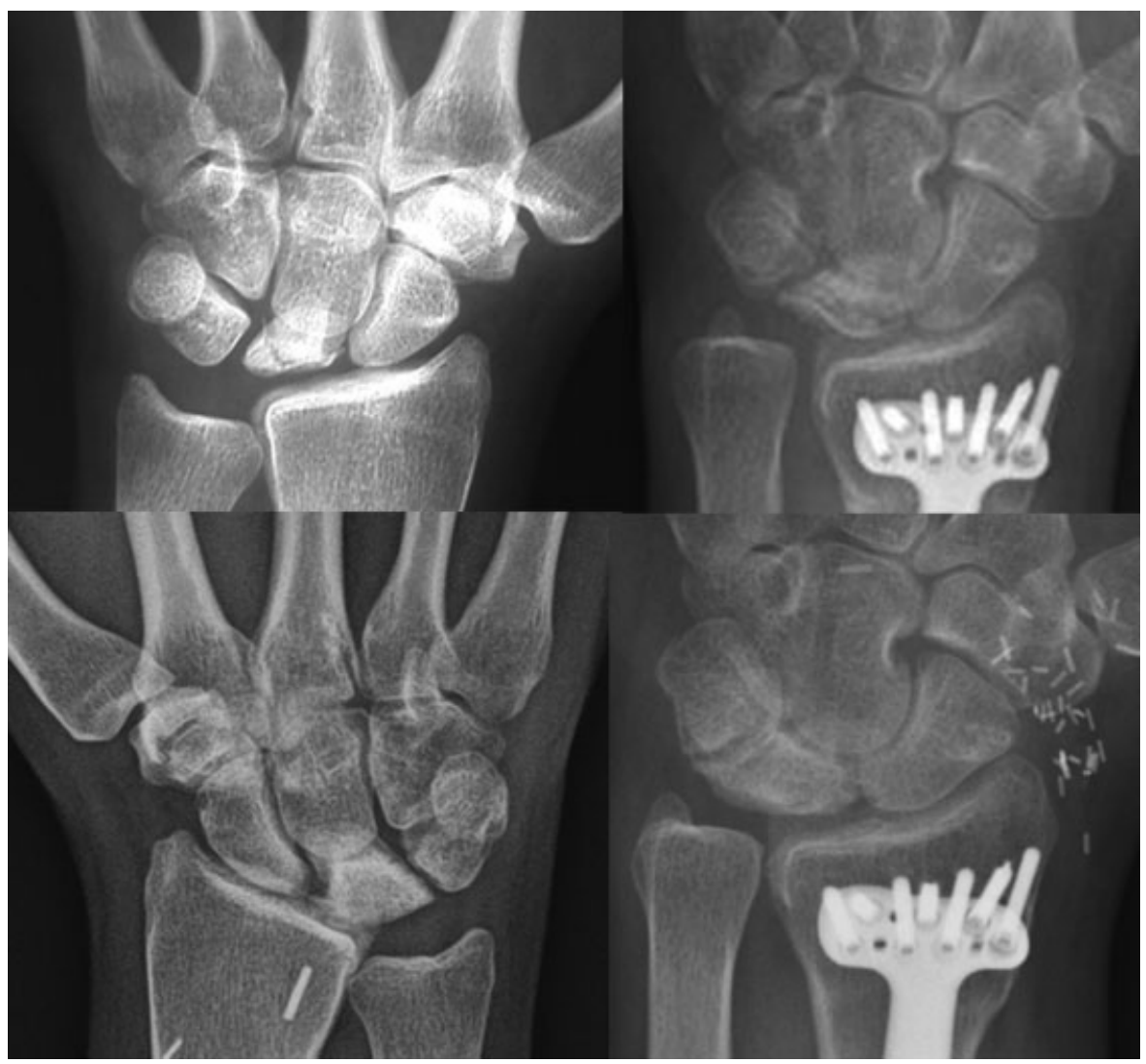

Fig. 4 Preoperative anteroposterior (AP) (above) and postoperative AP and lateral X-rays (below) of cases of advanced Kienböck disease lunate medial femoral trochlea (MFT) reconstructions. On the left is a 30-year-old patient with stage 3A Kienböck disease without scaphoid flexion or radiocarpal arthritis (top left). At 19.5 months after MFT reconstruction (bottom left), she has achieved improved carpal height, Stahl Index, and lunate substance, converting to stage 2 disease. On the right is 23 -year-old patient with stage 3A Kienböck disease without scaphoid flexion or radiocarpal arthritis. Initial treatment with radial shortening osteotomy provided pain relief until he experienced recurrence of pain and coronal fracture of lunate (top right). Because of progression of the disease, MFT reconstruction via dorsal approach was performed. At 13 months after surgery, he has achieved improved carpal height and lunate substance, converting to stage 2 disease and is pain free (bottom right).

All but one patient reported pain relief at 1 year, with $75 \%$ of the patients reporting complete pain relief. All but one patient demonstrated CT confirmed healing of the reconstructed lunates ( - Fig. 4). One patient required revision to a total wrist arthrodesis. Lichtman radiographic staging of Kienböck improved in four patients from a $3 \mathrm{~A}$ to a 2 at $1-$ year follow-up. In 10 patients, the Lichtman stage remained unchanged (but did not progress), and in the remaining 2 patients, the Lichtman stage progressed from $3 \mathrm{~A}$ to $3 \mathrm{~B}$ but did not require further surgery.

\section{Other Applications: Capitate Resurfacing}

The MFT cartilage is also noted to be of similar arc of curvature to the proximal aspect of the capitate. This has enabled the authors to reconstruct a rare case of capitate avascular necrosis (AVN) (one patient) (-Fig. 5), and resurface the arthritic proximal capitate to provide a motion sparing alternative to wrist fusion in patients with stage 4 Kienböck disease and loss of capitate cartilage (four patients) performed in conjunction with a proximal row carpectomy.

\section{Other Applications: Talus Resurfacing}

The MFC bone flap has been described for revascularization of AVN of the talus. ${ }^{9}$ Similar to Kienböck disease and collapse, the reconstruction of the talus can also be performed with cartilage-bearing flaps to provide stout structural reconstruction of the medial talus with a similarly convex lateral trochlea of the femur (- Fig. 6). Here, the periosteal vessels are supplied by the SLGA. The pedicle is shorter than the DGA on the medial femur, but it is of generous caliber and easy to locate. The anastomosis into the posterior tibial artery is feasible without grafting because of its proximity to the surgical site.

\section{Other Applications: Radiocapitellar Joint Resurfacing}

In the setting of radiocapitellar joint pain in posttraumatic arthritis in younger patients, the senior author (H.K.B.) has performed osteochondral resurfacing of either the capitellum or the capitellum and radial head articular surfaces (five cases). The posterior medial aspect of the knee joint is 

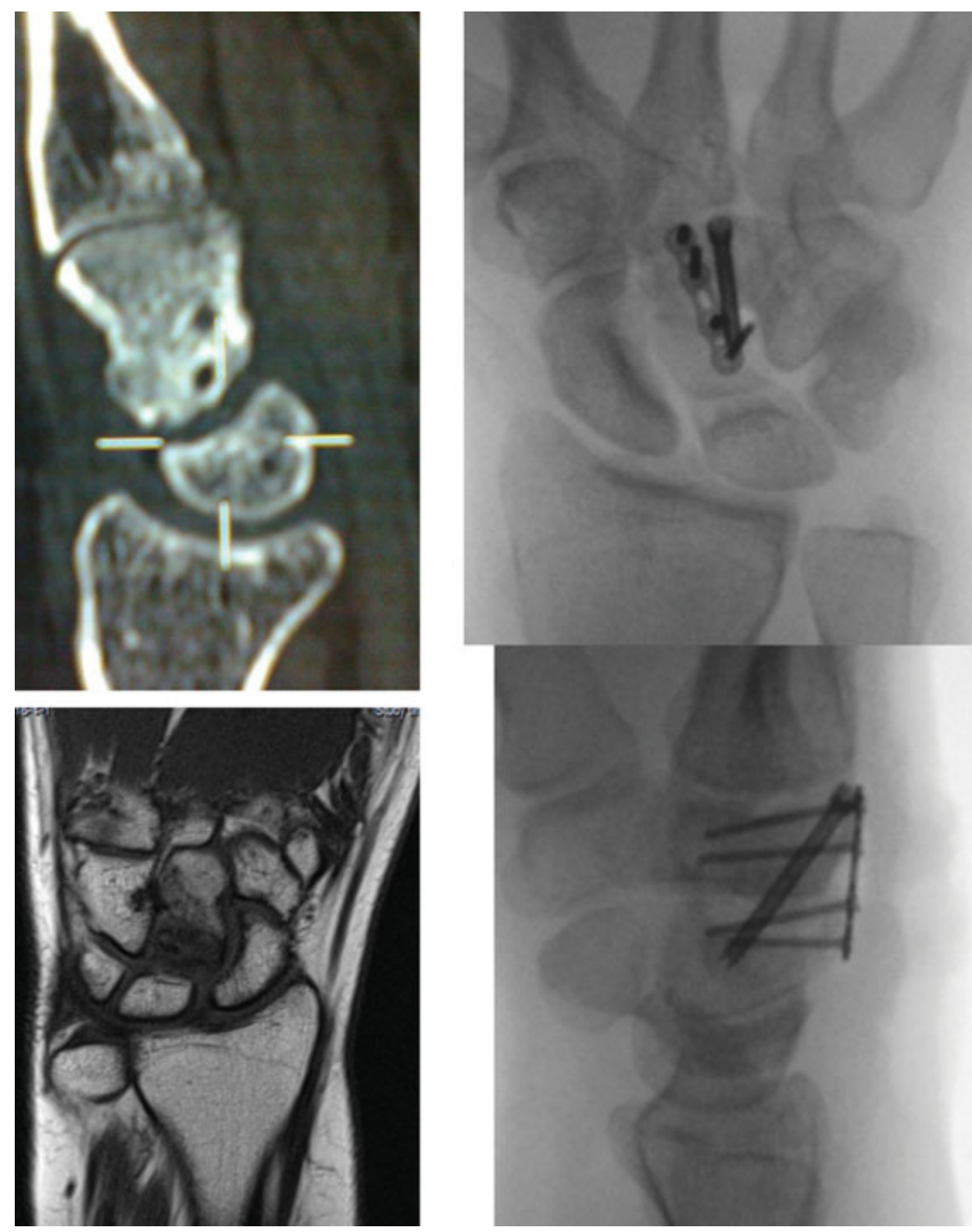

Fig. 5 Example of medial femoral trochlea (MFT) reconstruction of proximal capitate. A 28-year-old patient with spontaneous avascular necrosis $(A V N)$ of proximal capitate. Top left, preoperative lateral X-ray demonstrating proximal capitate destruction with well-preserved distal lunate cartilage. Bottom left, preoperative magnetic resonance imaging showing avascular necrosis changes limited to proximal half of capitate. Top right, postoperative anteroposterior X-ray after MFT reconstruction with plate and screw fixation. Bottom right, postoperative lateral X-ray of reconstructed capitate.

explored and the proximal most segment of posterior femoral cartilage is harvested based on a periosteal branch from the DGA system. This concave curvature is utilized for the capitellum reconstruction. If the radial head is also to be reconstructed, the shallow convex surface of the medial patella is harvested on a DGA branch coursing through the medial capsule enabling the two segments to be harvested as a chimeric osteochondral flap (-Fig. 7).

\section{Summary}

The use of vascularized cartilage flaps from the distal femur holds promise because of the morphological similarity to articular surfaces involved in challenging degenerative joint defects around the peripheral skeleton. Our growing experience with microvascular transfer of periosteal-based vessels may provide a basis for addressing many unsolved orthopedic problems with autologous tissue. Much investigation is still required to elucidate the natural history of cartilage survival in an intrasynovial environment with periosteal blood supply. Further work on the histologic performance of these flaps is underway, as well as examination of the microvascular network of periosteum throughout the skeleton. Finally, long-term follow-up on the donor site morbidity both radiographically and subjectively is required. 

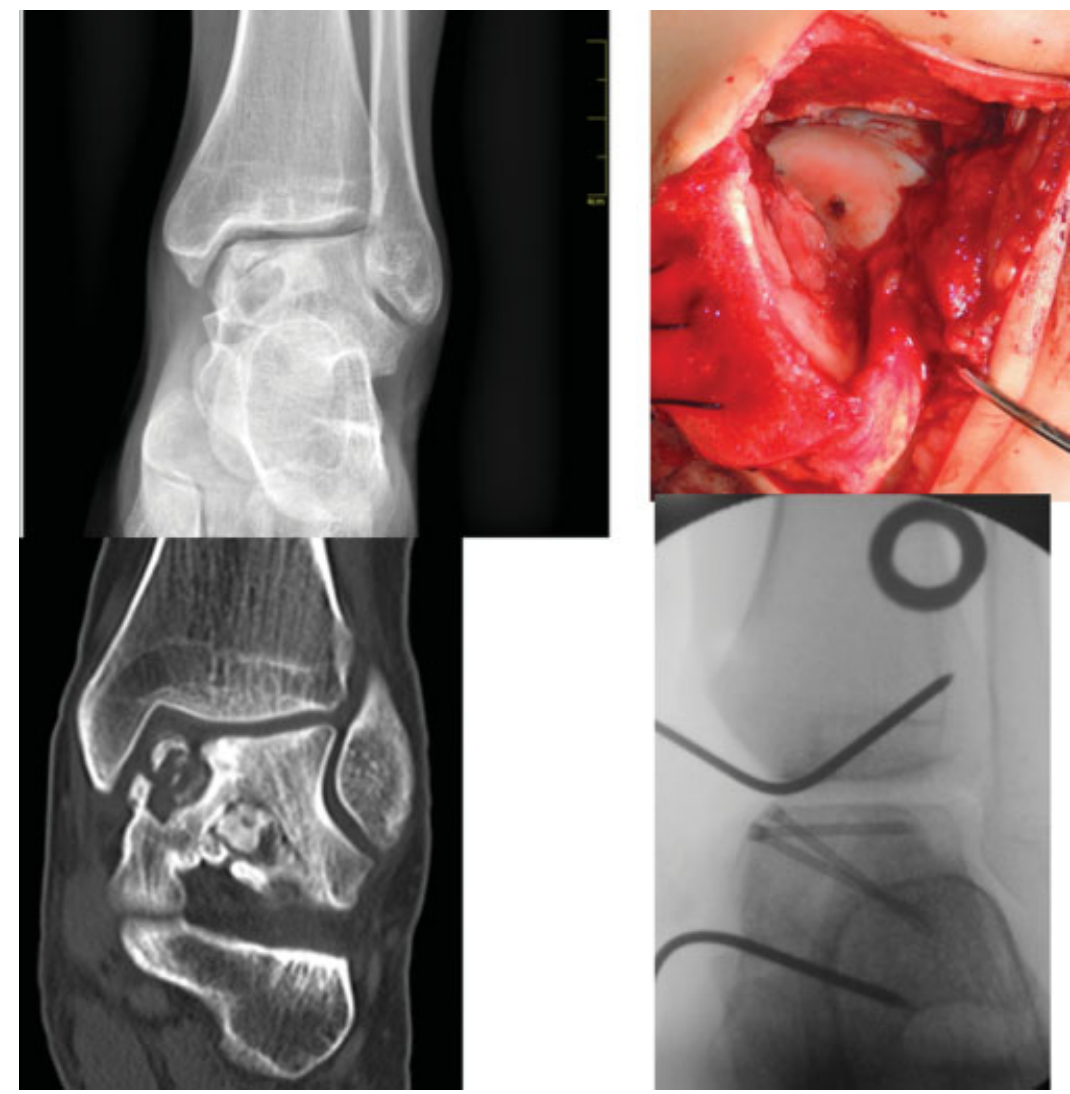

Fig. 6 A 18-year-old patient with avascular necrosis of the talus involving the proximal medial margin. Top left, preoperative anteroposterior (AP) X-ray. Bottom left, preoperative coronal computed tomographic scan. Top right, inset of lateral femoral trochlea to reconstruct the proximal medial osteochondral aspect of the talus. Bottom right, postoperative AP radiograph of the ankle demonstrating congruency of the reconstructed joint.
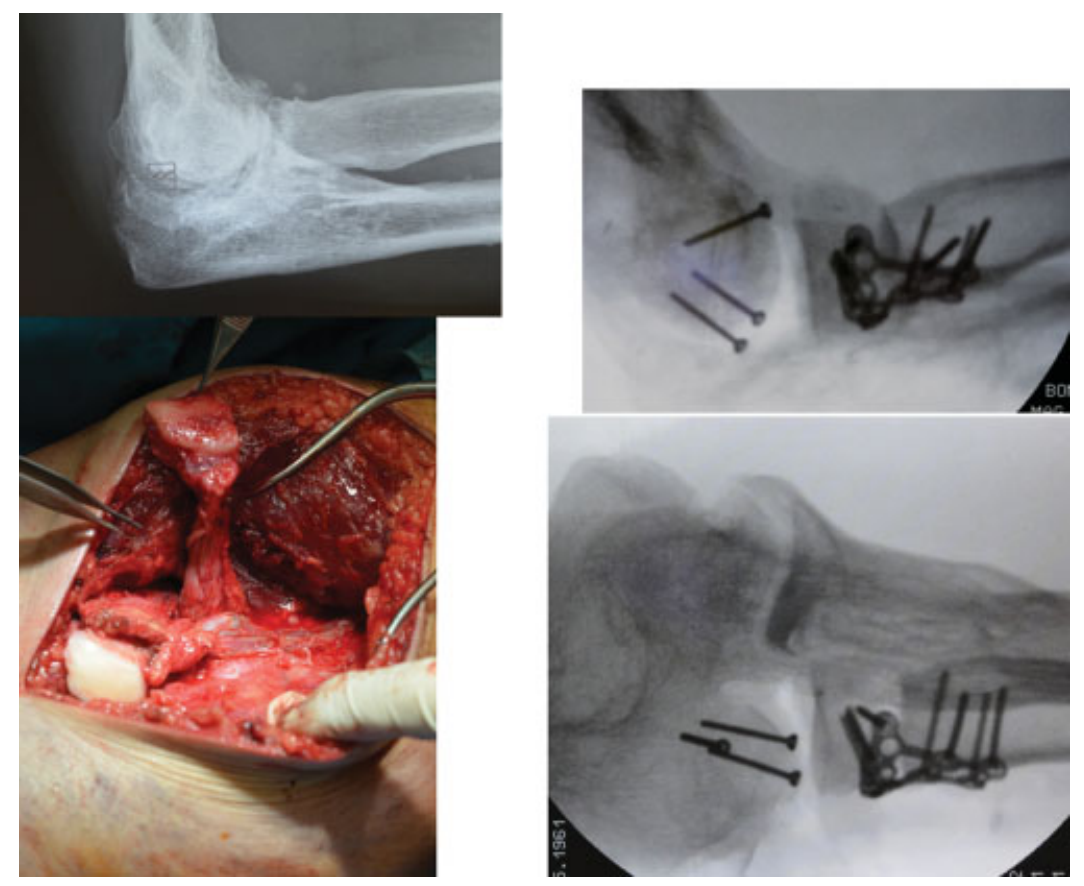

Fig. 7 A 42-year-old patient with devastating posttraumatic arthritis of the elbow complaining primarily of lateral elbow pain. Top left, poreoperative lateral X-ray of the elbow. Bottom left, elevation of two chimeric osteochondral components. The forceps in the top of the image are holding a shallowly concave segment of the medial patella attached via a leash of knee capsule housing branches from the descending geniculate artery (DGA) pedicle. The inferior cartilage segment is elevated from the proximal most segment of the posteromedial aspect of the femur. These are both attached via vascular leash to the DGA pedicle. Thigh is to the left and foot to the right. Right, postoperative lateral (top) and AP (bottom) X-ray of the elbow showing congruency of reconstructed radiocapitellar joint. 


\section{References}

1 Martin D, Bitonti-Grillo C, De Biscop J, et al. Mandibular reconstruction using a free vascularised osteocutaneous flap from the internal condyle of the femur. Br J Plast Surg 1991;44(6):397-402

2 Yamamoto H, Jones DB Jr, Moran SL, Bishop AT, Shin AY. The arterial anatomy of the medial femoral condyle and its clinical implications. J Hand Surg Eur Vol 2010;35(7):569-574

3 Iorio ML, Masden DL, Higgins JP. The limits of medial femoral condyle corticoperiosteal flaps. J Hand Surg Am 2011;36(10): 1592-1596

4 Hugon S, Koninckx A, Barbier O. Vascularized osteochondral graft from the medial femoral trochlea: anatomical study and clinical perspectives. Surg Radiol Anat 2010;32(9):817-825

5 Bürger HK, Windhofer C, Gaggl AJ, Higgins JP. Vascularized medial femoral trochlea osteocartilaginous flap reconstruction of proximal pole scaphoid nonunions. J Hand Surg Am 2013;38(4):690-700
6 Burger HK, Windhofer C, Gaggl AJ, Higgins JP. Vascularized medial femoral trochlea osteochondral flap reconstruction of advanced Kienböck disease. J Hand Surg Am (in press).

7 Kälicke T, Bürger H, Müller EJ. [A new vascularized cartilaguebone-graft for scaphoid nonunion with avascular necrosis of the proximal pole. Description of a new type of surgical procedure]. Unfallchirurg 2008;111(3):201-205

8 Iorio ML, Masden DL, Higgins JP. Cutaneous angiosome territory of the medial femoral condyle osteocutaneous flap. J Hand Surg Am 2012;37(5):1033-1041

9 Higgins JP, Bürger HK. Proximal scaphoid arthroplasty using the medial femoral trochlea flap. J Wrist Surg 2013;2(3): 228-233

10 Haddock NT, Alosh H, Easley ME, Levin LS, Wapner KL. Applications of the medial femoral condyle free flap for foot and ankle reconstruction. Foot Ankle Int 2013;34(10):1395-1402 